\title{
The Fuzzy Parametrized Model for Classifying Blocks in the Non-binary Motion Mask
}

\author{
Dmitry A. Matsypaev ${ }^{1}$ and Andrey G. Bronevich ${ }^{2}$ \\ 1 Southern Federal University,Taganrog Institute of Technology, Taganrog, Russia \\ 2 National Research University "Higher School of Economics", Moscow, Russia \\ \{dmitry.matsypaev, andreybronevich\}@gmail.com
}

\begin{abstract}
The motion detection in video is considered. We break nonbinary motion mask on blocks and calculate a certain statistics for each block. Then we use prior information about statistics distribution to classify blocks on background and foreground. The estimation framework for classification confidence is presented.
\end{abstract}

Keywords: non-binary motion mask, block representation, fuzzy model, imprecise probabilities.

\section{Introduction}

This paper tackles the problem of automatic detection of moving objects in the video sequence taken from the stationary monocular camera with the fixed focal length. Existing methods to solve it are described in detail in [1], [2, [5], [7], [8], 10], 11] etc.

We propose to build non-binary motion mask and to break it on rectangular blocks. For each block we estimate the special statistics whose distribution allows us to decide, whether a certain block belongs to foreground or to background. We introduce the fuzzy mathematical model of classification that is based on the prior information of how moving objects appear in the video sequence.

\section{Building the Motion Mask}

Let us assume that we have the sequence of video frames $\left\{F^{(k)}\right\}$, where each frame is a gray scale image with the $W_{F} \times H_{F}$ resolution. In [1], [2], [5], [7], [8], [10, [11] the motion mask $M^{(k)}$ for the frame $F^{(k)}$ is a binary image derived from the frame set $\left\{F^{(k-m+1)}, \ldots, F^{(k)}\right\}, m>1$. To build a binary mask, the threshold transformation can be used. Applied to the signal, we can loose some useful information about motion objects, therefore, in the paper we suggest to use the non-binary motion mask. The simplest way to obtain it is to compute the absolute difference between two neighboring frames without threshold transformation:

$$
M_{i j}^{(k)}=\left|F_{i j}^{(k)}-F_{i j}^{(k-1)}\right|
$$

where $1 \leq i \leq W_{F}, 1 \leq j \leq H_{F}, 0 \leq M_{i j}^{(k)} \leq I_{\max }$, and $I_{\max }$ is the maximal value of the pixels intensity. 


\section{Representation of Blocks in the Motion Mask}

The representation of images with the help of blocks is widely used in video coding [9] and image processing [4, [8]. In this case we break each motion mask on rectangular blocks with constant size $W_{B} \times H_{B}$. Let $U$ be the set of such blocks. For each block $B \in U$ the following statistics is computed:

$$
I(B)=\frac{1}{W_{B} H_{B}} \sum_{i=1}^{W_{B}} \sum_{j=1}^{H_{B}} B_{i j},
$$

where $B_{i j}$ is the pixel intensity value for block $B$ at column $i$ and row $j$. Such a motion mask representation fits the localization and compactness properties of moving areas, reduces computational complexity and decreases the effect of noise. However, the geometrical precision of the result is bounded from below by the size of the block.

\section{Foreground Blocks Detection}

\subsection{Basic Hypotheses}

We will describe next the properties of motion blocks with the help of two fuzzy sets: by a fuzzy set of foreground blocks $U_{f g}$ with the membership function $\mu_{f g}(B)$ and by a fuzzy set of background blocks $U_{b g}$ with the membership function $\mu_{b g}(B)$, where $B \in U$. Let us assume that these sets obey the following hypothesis.

Hypothesis 1. For all $B_{1}, B_{2} \in U$ the statement $I\left(B_{1}\right) \geq I\left(B_{2}\right)$ is true if and only if $\mu_{b g}\left(B_{1}\right) \leq \mu_{b g}\left(B_{2}\right)$ and $\mu_{f g}\left(B_{1}\right) \geq \mu_{f g}\left(B_{2}\right)$.

The Hypothesis 1 states that the statistics (2) can be used to order the elements of the set $U$ by the membership functions values. Assuming that the Hypothesis 1 is true, let us postulate the additional two hypotheses.

Hypothesis 2. Let $C_{b g}=\left\{B: \mu_{b g}(B)=1\right\}$. Then $\left|C_{b g}\right| /|U|=\alpha$, where $\alpha \in$ $(0,1)$ is the parameter of the hypothesis.

Hypothesis 3. Let $C_{f g}=\left\{B: \mu_{f g}(B)=1\right\}$. Then $\left(I_{\max }-\min _{B \in C_{f g}} I(B)\right) /$ $I_{\max }=\beta$, where $\beta \in(0,1)$ is the parameter of the hypothesis.

Hypotheses 2 and 3 are derived from the analysis of a typical plot of the cumulative distribution function computed for the statistics (2)

$$
F_{I}(x)=\frac{1}{|U|} \sum_{B \in U \mid I(B) \leq x} 1 .
$$

As you can see on Fig. 1 for the most of blocks the statistics $I$ is close to zero while for the minority of blocks $I$ is dispersed over the segment $\left[0, I_{\max }\right]$. It means that both $\alpha$ and $\beta$ should be close to 1 . We set these parameters to 0.9 in our experiments. 


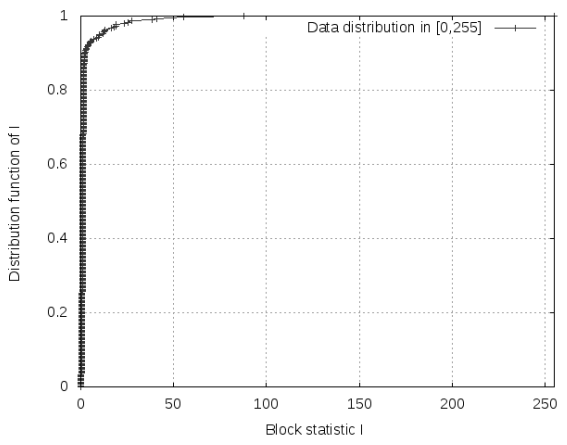

Fig. 1. The typical plot for $F_{I}$

\subsection{Membership Function Formalization}

Let us introduce the value $I_{\alpha}=\min _{x \in\left[0, I_{\max }\right]}\left\{x \mid F_{I}(x) \geq \alpha\right\}$. Then the formalization of membership function $\mu_{b g}(B)$ based on Hypotheses 1 and 2 leads to the following. Let $\mu_{b g}(B)=\eta_{b g}(I(B))$, where $\eta_{b g}(x)$ satisfies the following conditions: 1) $\eta_{b g}(x)$ is defined on $\left.\left.\left[0, I_{\max }\right], 2\right) \forall x \in\left[0, I_{\alpha}\right] \eta_{b g}(x)=1,3\right) \eta_{b g}\left(I_{\max }\right)=0$ and 4) $\eta_{b g}(x)$ is monotone and non-increasing on $\left[0, I_{\max }\right]$.

Analogously, let us introduce the value $I_{\beta}=(1-\beta) I_{\max }$. Then the formalization of membership function $\mu_{f g}(B)$ based on Hypotheses 1 and 3 leads to the following. Let $\mu_{f g}(B)=\eta_{f g}(I(B))$, where $\eta_{f g}(x)$ satisfies the following conditions: 1) $\eta_{f g}(x)$ is defined on $\left.\left.\left[0, I_{\max }\right], 2\right) \forall x \in\left[I_{\beta}, I_{\max }\right] \eta_{f g}(x)=1,3\right)$ $\eta_{f g}(0)=0$ and 4$) \eta_{f g}(x)$ is monotone and non-decreasing on $\left[0, I_{\max }\right]$.

\subsection{Tversky's Functional}

The parametrized Tversky's functional is used in fuzzy set theory to estimate the degree of similarity of two sets 3 :

$$
T_{a, b}(X, Y)=\frac{f(X \cap Y)}{f(X \cap Y)+a f(X-Y)+b f(Y-X)},
$$

where $X, Y$ is a pair of fuzzy sets, $\cup, \cap,-$ are consistent operations to unite, intersect and subtract two fuzzy sets, $f$ is a functional that satisfies $f(X \cup Y)=$ $f(X)+f(Y)$ for all disjoint sets $X, Y$, and $a, b \in \mathbb{R}$ are parameters.

It is possible to prove that functions $\eta_{b g}$ and $\eta_{f g}$ satisfy the conditions proposed above if they are defined as

$$
\eta_{b g}(x)=\left(\phi\left(x, I_{\alpha}\right)-\phi\left(I_{\max }, I_{\alpha}\right)\right) /\left(1-\phi\left(I_{\max }, I_{\alpha}\right)\right),
$$

where

$$
\phi(x, y)=T_{1 / 2,0}([-\gamma / 2, x],[-\gamma / 2, y])=\frac{2 \min \{x, y\}+\gamma}{2 \min \{x, y\}+\gamma+\max \{x-y, 0\}},
$$


and

$$
\eta_{f g}(x)=\left(\psi\left(x, I_{\beta}\right)-\psi\left(0, I_{\beta}\right)\right) /\left(1-\psi\left(0, I_{\beta}\right)\right)
$$

where

$$
\psi(x, y)=T_{0,1 / 2}([-\gamma / 2, x],[-\gamma / 2, y])=\frac{2 \min \{x, y\}+\gamma}{2 \min \{x, y\}+\gamma+\max \{y-x, 0\}}
$$

The calibration parameter $\gamma>0$ is chosen such that

1. $\phi(0,0)=1$;

2. for fixed $x_{0} \in\left(0, I_{\max }\right]$ and $\phi_{0} \in(0,1)$ the statement $\phi\left(x_{0}, 0\right)=\phi_{0}$ is true.

We used $x_{0}=1$ and $\phi_{0}=2 / 3$ in our experiments. If $I_{\max }=255$, then $\gamma=2$.

\subsection{Block Classification}

To determine whether a block is foreground or background, we need to check inequality

$$
\mu_{f g}(B) \geq \mu_{b g}(B) .
$$

We define below the probability $p_{f g}(B)$ of block $B \in U$ to be in the foreground using the model of imprecise probabilities [6]. In this case the lower probability is defined as $\underline{p}_{f g}(B)=\min \left\{\mu_{f g}(B), 1-\mu_{b g}(B)\right\}$ and the upper probability is defined as $\bar{p}_{f g}(B)=\max \left\{\mu_{f g}(B), 1-\mu_{b g}(B)\right\}$. Such estimates are not contradictory because $\left[\underline{p}_{f g}(B), \bar{p}_{f g}(B)\right]$ is a convex set $[\underline{6}$.

We take a mean point of the set above as the expected foreground probability value $p_{f g}(B)$. Obviously,

$$
p_{f g}(B)=0.5\left(\mu_{f g}(B)+1-\mu_{b g}(B)\right) .
$$

The equation (4) allows us to define a more general criterion comparing to (3):

$$
p_{f g}(B) \geq p_{t h},
$$

where $p_{t h} \in[0,1]$ is a threshold parameter. Let us notice that inequality (3) is equivalent to (5) if $p_{t h}=1 / 2$.

\subsection{Confidence Estimation}

The use of imprecise probabilities allows us to estimate the confidence of classification in bits. Let a block $B$ be classified then we can estimate the distrust level using the formula below:

$$
D(B)=-p_{\text {med }} \log _{2} p_{\text {med }}-\left(1-p_{\text {med }}\right) \log _{2}\left(1-p_{\text {med }}\right),
$$

where $p_{\text {med }}=\operatorname{median}\left\{\bar{p}_{f g}(B), \underline{p}_{f g}(B), 1 / 2\right\}$. The distrust level depends on the block statistics $I$ as shown on Fig 2 Its distribution is unimodal with a high peak where statistics $I$ has intermediate values. 


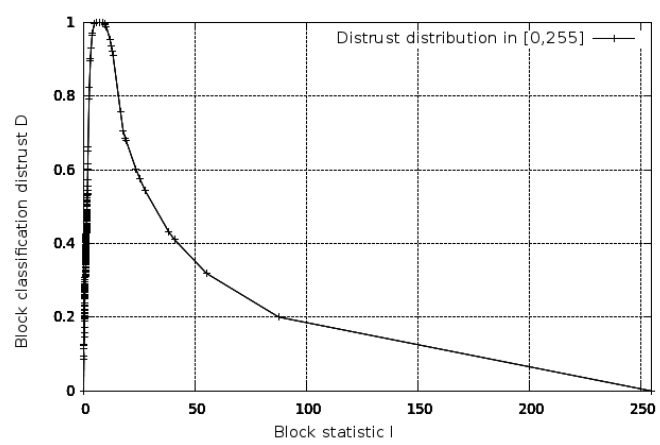

Fig. 2. The dependence between block statistics $I$ and estimated distrust level $D$

\section{$5 \quad$ Experiments}

To proceed the quality evaluation, we have manually labeled 3 video sequences. During the experiments we set the size of blocks to $16 \times 16$ and the parameter $p_{t h}$ to 0.5 For the comparison we have chosen the Mixture of Gaussians (MoG) algorithm [11] implemented in the OpenCV programming library 2]. It is an ancestor of a lot of more specialized and slow state-of-the-art techniques which are adapted to specific cases [1].

On the Fig. 3 one can see the ROC-curve which compares the presented algorithm and the MoG. It should be noted that our algorithm bounds the precision from below because it operates with each the block containing 256 pixels as the whole. That's why MoG superiors in the $[0.13 ; 0.97]$ segment of the True Positive Rate. As for the performance evaluation, our algorithm is faster than MoG 2-5 times depending on a certain video.

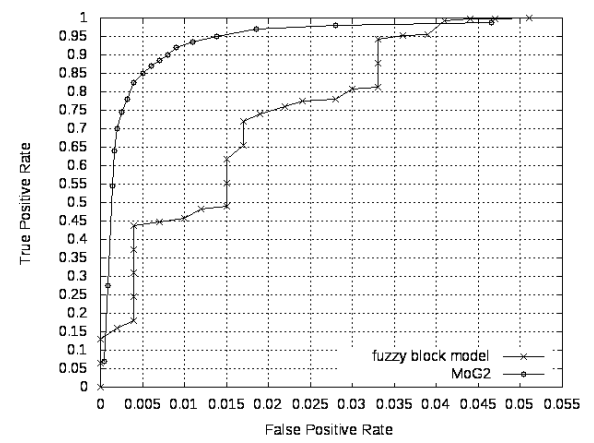

Fig. 3. The ROC-curve to compare presented algorithm (aka fuzzy block model) with Mixture of Gaussians (aka MoG2) 


\section{Conclusion}

The approach of detecting moving objects in the video sequence is presented. The main idea is to construct the non-binary motion mask and to use its block representation for further analysis. The latter is based on the mathematical model that formalizes prior information and hypotheses obtained during the proceeded experiments. The mathematical framework to estimate the confidence of motion mask segmentation is considered. Further research will be dedicated to choosing the optimal parameters of the model presented above and to constructing moving areas from the foreground blocks.

\section{References}

1. Bouwmans, T.: Recent advanced statistical background modelling for foreground detection: a systematic survey. Recent Patents on Computer Science 4(3), 147-176 (2011)

2. Bradski, G., Kaehler, A.: Learning OpenCV. Computer vision with the OpenCV library. O'Reilly Media, Inc., Sebastopol (2008)

3. Cross, V.V., Sudkamp, T.A.: Similarity and compatibility in fuzzy set theory: assessment and applications. Physica-Verlag GmbH \& Co., Heidelberg (2002)

4. Guaourova, A., Kamath, C., Cheung, S.C.: Block matching for object tracking. Technical report, Lawrence Livermore National Laboratory (2003)

5. Hofmann, M., Tiefenbacher, P., Rigoll, G.: Background segmentation with feedback: the pixel-based adaptive segmenter. In: Proc. of IEEE Workshop on Change Detection, pp. 38-43 (2012)

6. Klir, G.J.: Uncertainty and information. Foundations of generalized information theory. Jonh Wiley \& Sons, Inc., New York (2006)

7. Narayana, M., Hanson, A.R., Learned-Miller, E.G.: Background modelling using adaptive pixelwise kernel variances in a hybrid feature space. In: CVPR, pp. 2104-2111. IEEE (2012)

8. Park, J., Tabb, A., Kak, A.C.: Hierarchical data structure for real time background subtraction. In: IEEE International Conference on Image Processing, pp. 1849-1852. IEEE, Atlanta (2006)

9. Richardson, I.E.G.: H.264 and MPEG-4 video compression: video coding for next generation multimedia. Jonh Wiley \& Sons Ltd., Chischester (2003)

10. Van Droogenbroeck, M., Paquot, O.: Background subtraction: experiments and improvements for ViBe. In: Proc. of 2012 IEEE Computer Society Conference, pp. 32-37. Providence (2012)

11. Zivkovic, Z.: Improved adaptive Gaussian mixture model for background subtraction. In: International Conference Pattern Recognition, vol. 2, pp. 28-31. ICPR, Cambridge (2004) 